\title{
A Rare Case of Ovarian Follicular Lymphoma: Incidental Finding in a Woman With Postmenopausal Bleeding
}

\author{
Caroline Chiew Ping Tan ${ }^{\mathrm{a}, \mathrm{d}}$, Yen Ching Yeo $^{\mathrm{b}}$, Ieera Madan Aggarwal ${ }^{\mathrm{c}}$, Manisha Mathur
}

\begin{abstract}
Ovarian lymphoma is an uncommon malignancy with the incidence of primary ovarian lymphoma being ever rarer. Majority of patients with ovarian lymphoma tend to present with symptoms of abdominal distension, abdominal pain, loss of weight or appetite, and are usually found to have an ovarian mass. Postmenopausal bleeding (PMB) is not a symptom that is usually associated with ovarian pathology. This case report gives an account of how the patient presented to us, and the investigations and treatments offered that led to the final outcome.
\end{abstract}

Keywords: Follicular lymphoma; Postmenopausal bleed; Ovarian lymphoma

\section{Introduction}

Ovarian follicular lymphoma is a rare type of lymphoma that affects the ovary. The most common presenting complaints for ovarian tumors are those of abdominal or pelvic mass, abdominal pain, loss of weight, etc. [1], which can be vague and non-specific [2]. Some ovarian tumors can present with postmenopausal bleeding (PMB), but uterine pathology should be assessed before proceeding with evaluation for ovarian cancer [3]. PMB is more often associated with endometrial cancer, approximately 6-19\% [3]. Atypical presentation with heavy menstrual bleeding may also occur de novo or secondary to disseminated disease, with ovarian tumors [4]. Our patient here instead presented with symptoms of PMB alone with no gross abnormality of the ovaries on the pelvic scan. We would like to share the experience of managing such a case with an incidental diagnosis of ovarian follicular lymphoma on histology.

Manuscript submitted October 20, 2019, accepted November 12, 2019

aDepartment of Obstetrics and Gynaecology, KK Women's and Children's Hospital, Singapore 229899, Singapore

bDepartment of Pathology and Laboratory Medicine, KK Women's and Children's Hospital, Singapore 229899, Singapore

'Department of Gynaecology Oncology, KK Women's and Children's Hospital, Singapore 229899, Singapore

${ }^{\mathrm{d} C}$ Corresponding Author: Caroline Chiew Ping Tan, Department of Obstetrics and Gynaecology, KK Women's and Children's Hospital, 100 Bukit Timah Road, Singapore 229899, Singapore. Email: caroline.tan@mohh.com.sg

doi: https://doi.org/10.14740/jmc3386

\section{Case Report}

A 52-year-old lady (para 1) presented to the gynecology clinic with a history of PMB. She had undergone menopause 2 years prior to the presentation. She described it as per vaginal spotting to heavier flow for 10 days. It recurred again after 1 month. There was no abdominal pain or other symptoms. She has a past history of thyroid nodules which she underwent thyroidectomy in 2013. She was hypothyroid since then on thyroxine. She was under regular follow-up with an endocrinologist and her last thyroid function test was normal. She was also a known asthmatic on Budamate inhaler. Her recent cervical smear and mammogram in 2017 was normal. She has a strong family history of malignancy in her paternal side which includes throat, rectal and stomach cancer while her aunt had breast cancer. She had a pelvic scan done which showed endometrial thickness of $8 \mathrm{~mm}$, no lesions in the endometrial cavity, multiple uterine fibroids ranging in sizes from 0.9 to $4.7 \mathrm{~cm}$. Both ovaries contained unilocular anechoic avascular cysts measuring 0.5 and $0.6 \mathrm{~cm}$ on the right and left side, respectively (Fig. 1a-d). She underwent hysteroscopy, dilatation and curettage for assessment of her PMB. Intraoperative findings showed a $2-\mathrm{cm}$ endometrial polyp which was removed uneventfully. Histology of the specimen showed endometrial stromal breakdown with no evidence of malignancy. In view of this, the plan was to observe and to report any further PMB. One month later, she presented with another episode of significant PMB lasting 3 days which she described as "like a normal period". In view of the recurrent episodes of PMB, the patient was advised a hysterectomy as a form of management. Magnetic resonance imaging (MRI) pelvis done prior to her hysterectomy showed multiple fibroids with no focal endometrial lesion. A $0.9-\mathrm{cm}$ hemorrhagic cyst in the left ovary likely to be functional was seen (Fig. 2). There was no lymphadenopathy. The patient underwent an uncomplicated total laparoscopic hysterectomy and bilateral salpingo-oophorectomy.

Intraoperative findings were that of a normal appearing pelvic anatomy, including the uterus and the adnexa. Histology of the hysterectomy specimen confirmed the presence of uterine leiomyomas and adenomyosis. A nodular lymphoid proliferation is seen in the mildly enlarged ovary in keeping with left ovarian follicular lymphoma (Fig. 3a, b), the World Health Organization (WHO) grades 2 and 3A. Atypical smallto-medium lymphoid infiltrate is also seen in the right ovary in which involvement by lymphoma cannot be excluded. In view of the rare and unusual histological findings, the case was dis- 

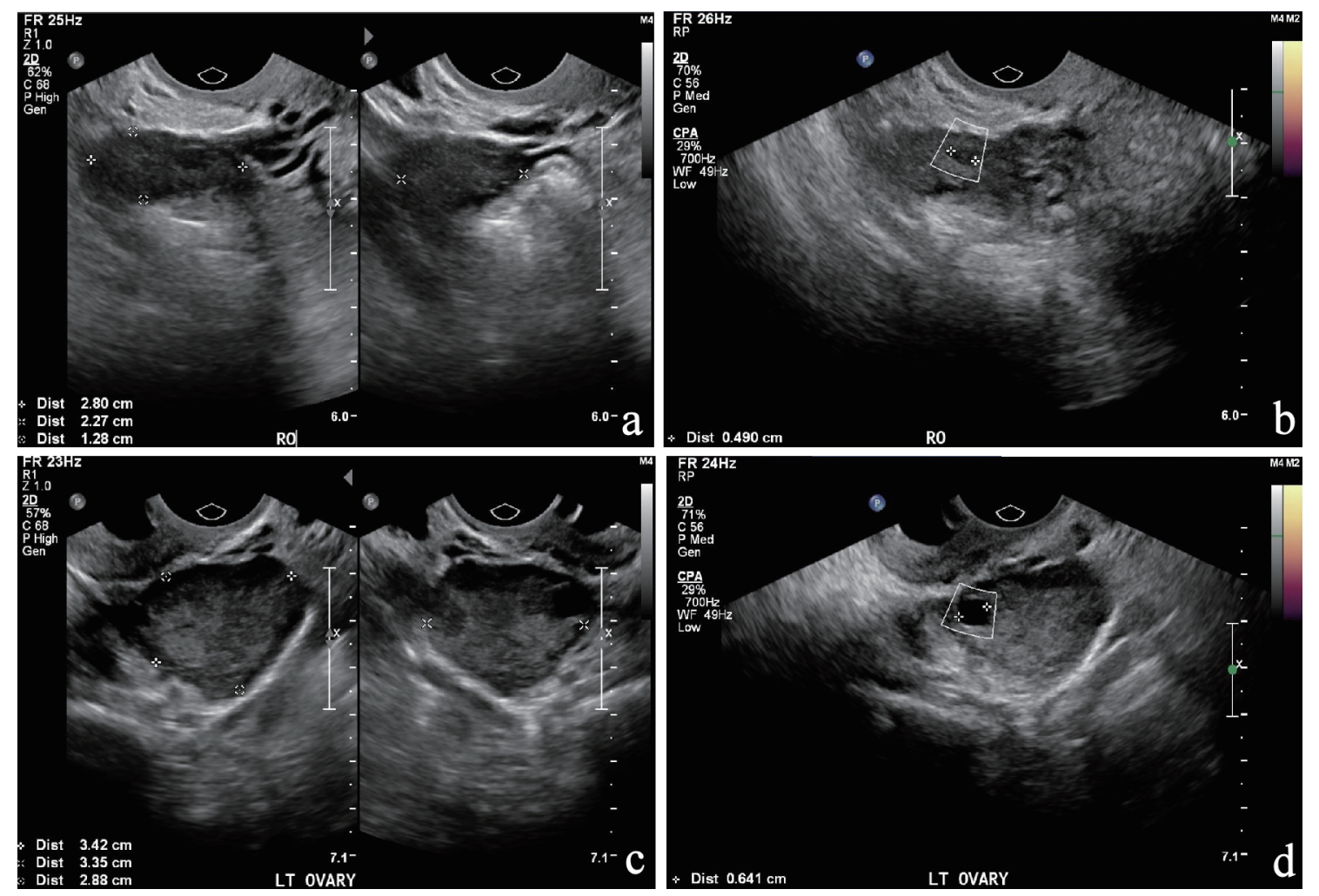

Figure 1. (a) and (b): US of pelvis of the right ovary; (c) and (d): US of pelvis of the left ovary. US: ultrasonography.

cussed at the local multidisciplinary team (MDT) meeting. The MDT recommended a positron emission tomography (PET) scan and referral to the lymphoma specialist. The PET scan showed no abnormal fluorodeoxyglucose (FDG)-avid nodes seen above and below the diaphragm, and no FDG-avid focus of disease detected. There was a superior mediastinal nodule known since thyroidectomy in 2013 which is of low-grade ac-

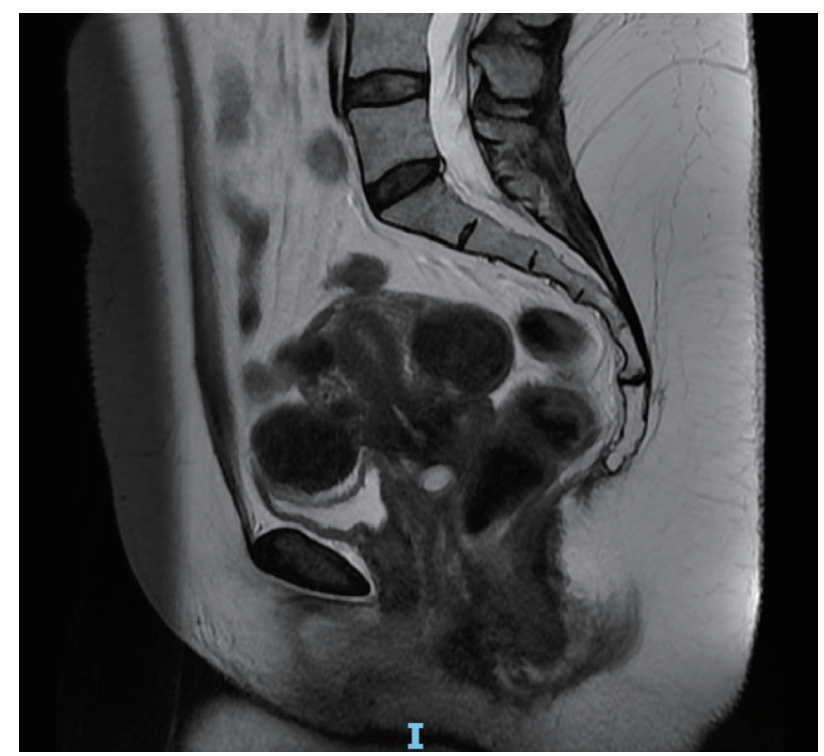

Figure 2. MRI of pelvis. MRI: magnetic resonance imaging. tivity on PET scan and is likely to be a remnant thyroid nodule. The management plan by her lymphoma specialist, following a detailed review is that of regular surveillance in view of the early stage of diagnosis and the results of the histology and PET scan.

\section{Discussion}

Lymphoma is a type of cancer that affects the white blood cells. Diagnosis of lymphoma is based on the WHO classification, and is divided into Hodgkin lymphoma (HL) and non-Hodgkin lymphoma (NHL) [5]. NHL is further classified into B-cell and T-cell types and subsequently into histologic subcategories $[5,6]$. The B-cell type NHL is the predominant type that can involve the genital tract including the ovary, uterus, cervix, vagina and the vulva [6]. The most common type is diffuse large B-cell lymphoma, which comprises approximately $25 \%$ of reported NHL cases in the West [7], with median age at diagnosis between 35 and 45 years while the follicular variety of lymphoma tends to occur in older women [8], as was the case with our lady. It is important to differentiate between primary or secondary lymphoma as it affects the prognosis and treatment. Primary ovarian lymphoma was defined as a lymphoma confined to ovary or primary extra nodal disease [1]. Secondary ovarian lymphoma was defined as secondary involvement of ovary in disseminated disease of nodal lymphoma [1,9]; and the distinction between primary verses secondary becomes difficult once it is disseminated [6]. 

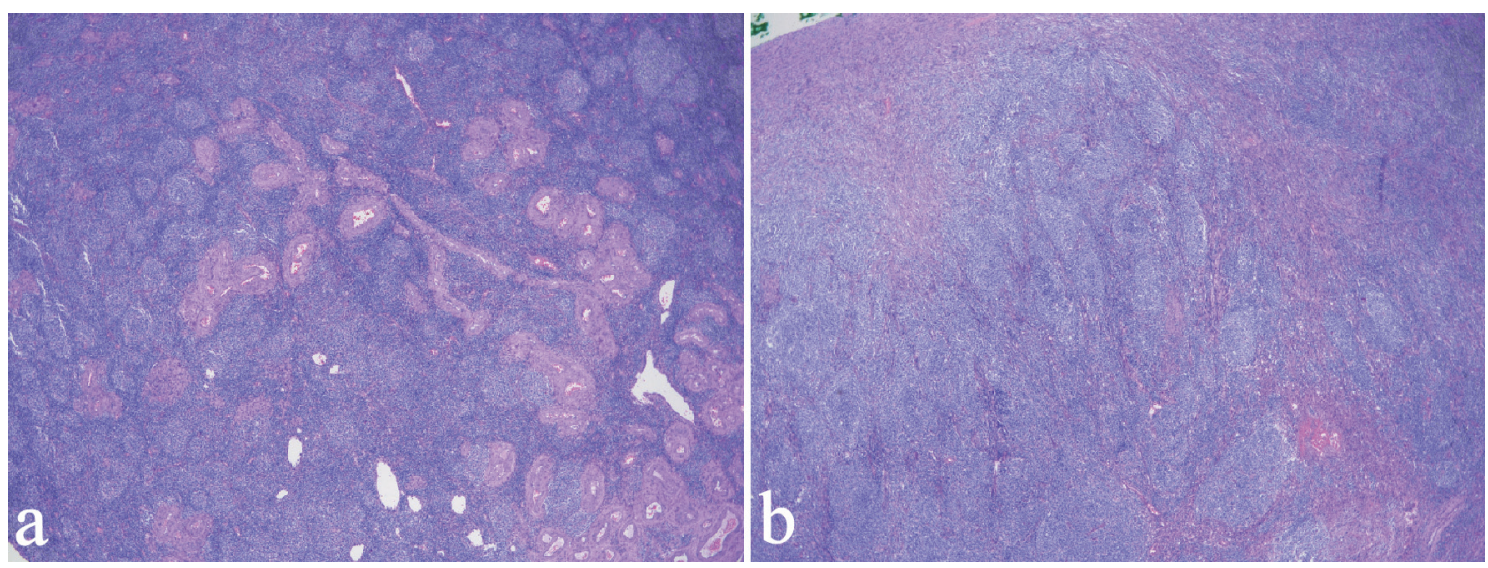

Figure 3. Low-power view of the ovarian follicular lymphoma. (a) Uniform nodularity with back to back arrangement of follicles showing mild variation in size and shape with no cell polarization and obvious tingible body macrophages. (b) A more "diffuse" appearance.

Ovarian NHL accounts for $1.5 \%$ among all the ovarian tumors and constitutes $0.5-1 \%$ among all NHL [1]. It frequently occurs as bilateral lesions especially in secondary cases [2]. Due to its rarity, there are only sporadic cases being reported in literature. The largest study in recent years by Ahmad and group published in 2014 showed that among the patients with ovarian lymphomas, $11 \%$ of the patients were asymptomatic, $24 \%$ patients presented with vague abdominal complaints and only $14 \%$ patients presented with constitutional symptoms [6]. Ovarian tumor markers such as cancer antigen 125 (CA 125) can be raised in primary gynecologic NHL, even though this marker has limited specificity [7]. Ovarian lymphomas are often difficult to differentiate from epithelial ovarian cancers or other type gynecological malignancies preoperatively, due to the lack of specific features related to lymphoma [6, 7]. Hence patients who present with adnexal masses should be treated like primary ovarian malignancy and explored surgically [7].

Immunohistochemical studies will aid in the subtyping, prognostication and treatment of NHL $[10,11]$. In our case, the left ovary is mildly enlarged and focally replaced by lymphoid

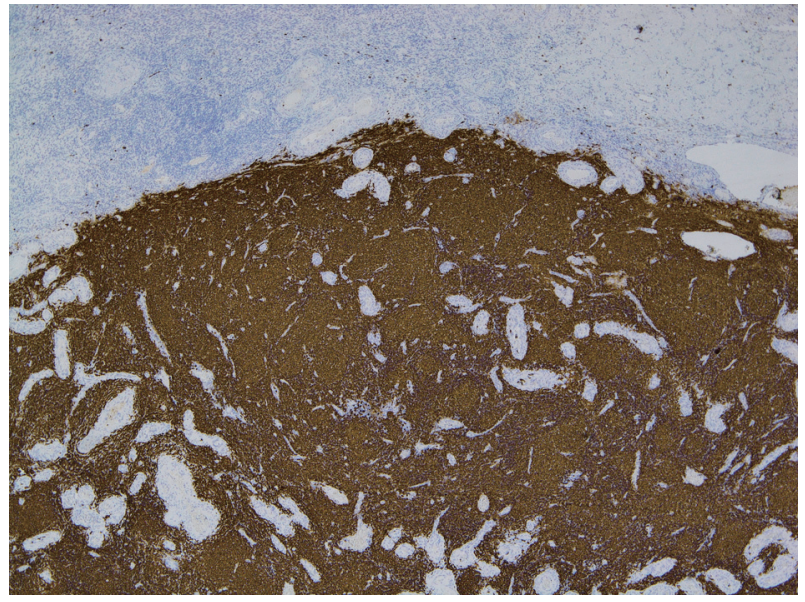

Figure 4. The lymphomatous proliferation strongly and diffusely expresses pan B-cell marker (CD 20). CD: cluster of differentiation. tissue with a follicular pattern. Lymphoid follicular proliferation was atypical and composed of a varying admixture of centrocytes and centroblasts, the latter numbering below 15 per high-power field of $0.159 \mathrm{~mm}^{2}$ in some areas, in keeping with WHO grade 2 follicular lymphoma, but exceeding this upper limit in others, commensurate with WHO grade $3 \mathrm{~A}$ follicular lymphoma. Lymphomatous proliferation strongly and confluently expressed pan B-cell marker cluster of differentiation (CD)20 (Fig. 4), while the follicle centers strongly expressing germinal center-associated B-cell lymphoma 6 (BCL-6) and CD10 (Fig. 5). The cell proliferation fraction in the neoplastic follicles on Ki-67 immunolabeling disclosed loss of normal polarity and was abnormally depressed but variable, ranging from $20 \%$ to $30 \%$ in grade 2 areas and culminating around $70 \%$ in grade $3 \mathrm{~A}$ follicles. The interfollicular compartment maintained a low proliferation fraction of approximately $5 \%$ to $10 \%$, hence not supporting a component of diffuse large cell lymphoma. Immunostaining for cyclin D1 was negative within the lymphomatous population, which excluded mantle cell lymphoma. Immunohistochemistry was carried out to confirm this.

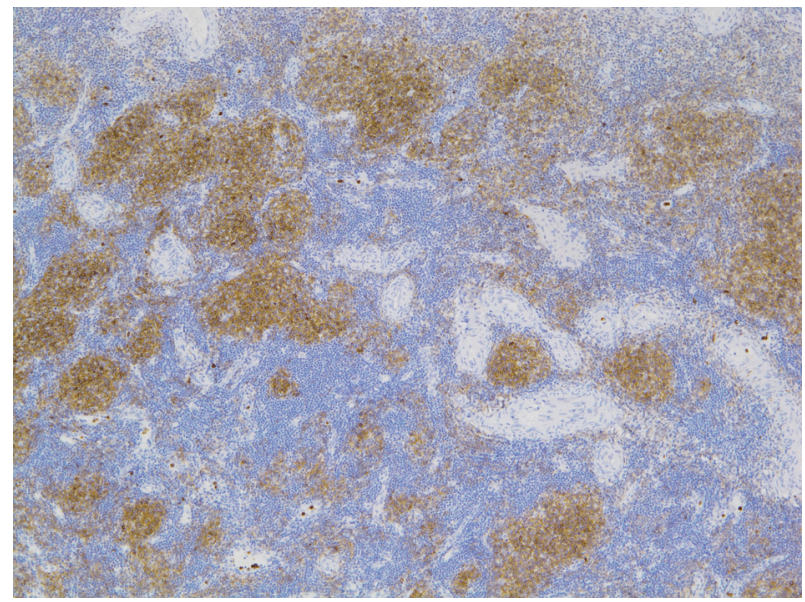

Figure 5. The follicles centers strongly express germinal centre-associated CD10. CD: cluster of differentiation. 
Once a diagnosis is confirmed, it is important to do lymphoma staging, and computed tomography (CT) scan of the chest, abdomen and pelvis can be used for this purpose. Bone marrow biopsy is also used for staging. PET scan is commonly used for staging, detecting recurrence and assessment of therapeutic response $[7,10]$. Prognosis for genital tract NHL can be variable and dependent on age, stage, primary versus disseminated, bilateral lesions, histology, raised lactate dehydrogenase (LDH) levels, etc. [6]. There were several reports which described treatment with surgical resection alone without adjuvant chemotherapy but only in patients with no evidence of disseminated disease and were disease free with long clinical follow-up [6]. Primary ovarian lymphoma tends to have a better outcome as it is less aggressive with 5-year survival rate of $80 \%$ [1]. Those with disseminated lymphoma with involvement of the ovaries have a poorer prognosis with a 5-year survival rate of $7-38 \%$ [12]. Patients who are diagnosed with ovarian lymphomas are generally treated with chemotherapy [13]. Rapidly proliferative NHL tumors are highly responsive to chemotherapy [7], and the first-line therapy includes cyclophosphamide, doxorubicin, vincristine, prednisone (CHOP) combined with rituximab (R) [7]. Slower growing or more indolent lymphoma subtypes do not require aggressive treatment such as CHOP-R. As NHL is very responsive to chemotherapy, radical gynecologic surgery can be avoided if the diagnosis is known earlier [7], i.e. biopsies of suspicious lesions preoperatively such as cervical/vaginal mass.

In our case, the histological diagnosis of ovarian lymphoma was an incidental finding after a hysterectomy for recurrent PMB with uterine fibroids. Her PET scan subsequent to the hysterectomy showed no other lymph node involvement. CA 125 was not done initially as there were no radiological proven adnexal masses preoperatively. LDH level was normal. Hence the long term was conservative with regular 6 months to 1 year surveillance. Her most recent review showed no evidence of recurrence and a repeat $\mathrm{CT}$ scan was planned in a couple of months.

\section{Conclusions}

Primary ovarian lymphoma is an extremely rare diagnosis but usually tends to be associated with an ovarian mass. Hence an initial gynecological assessment should include a thorough clinical assessment followed by appropriate imaging. If the presenting symptom is PMB only, as in our patient, further investigations including hysteroscopy and endometrial biopsy should be done to exclude uterine pathology. In the case of recurrent $\mathrm{PMB}$, as was the case with our lady, definitive treatment in the form of a hysterectomy should be considered, even in the presence of a normal CT or MRI scan to exclude rarer pathology; and this would be the most important learning point in this case. As otherwise, we may have missed diagnosing the ovarian follicular lymphoma in our patient at an early stage which could have affected the prognosis and long-term survival. Ovarian lymphoma is still a histological diagnosis made postoperatively, and it must be differentiated from other ovarian malignancies as its management and prognosis can differ.

\section{Acknowledgments}

None to declare.

\section{Financial Disclosure}

None to declare.

\section{Conflict of Interest}

None to declare.

\section{Informed Consent}

The consent was obtained prior to completing the case report.

\section{Author Contributions}

CCPT contributed to the writing of the manuscript. YCY, IMA, MM reviewed and adjusted the manuscript prior to submission.

\section{References}

1. Bhartiya R, Kumari N, Mallik M, Singh RV. Primary nonHodgkin's lymphoma of the ovary - a case report. J Clin Diagn Res. 2016;10(5):ED10-11.

2. Seidler SJ, Lovey PY, Busuioc CI, Huber DE. Gynaecological lymphomas: case reports and literature review of primary extranodal female genital tract and breast nonhodgkin lymphomas. Archives in Cancer Research. ISSN 2254-6081; 6(4):20

3. Chen LM, Berek JS, Goff B, Don S Dizon, Vanessa A Barss. Epithelial carcinoma of the ovary, fallopian tube, peritoneum: Clinical features and diagnosis. www.uptodate.com.

4. Ekanayake CD, Punchihewa R, Wijesinghe PS. An atypical presentation of an ovarian lymphoma: a case report. J Med Case Rep. 2018;12(1):338.

5. Higgins RA, Blankenship JE, Kinney MC. Application of immunohistochemistry in the diagnosis of nonHodgkin and Hodgkin lymphoma. Arch Pathol Lab Med. 2008;132(3):441-461.

6. Ahmad AK, Hui P, Litkouhi B, Azodi M, Rutherford T, McCarthy S, Xu ML, et al. Institutional review of primary non-hodgkin lymphoma of the female genital tract: a 33year experience. Int J Gynecol Cancer. 2014;24(7):12501255.

7. Vijayakumar S, Serur E, Bybordi B, Lakhi N. Primary gynecological non-Hodgkin's lymphoma: A differential diagnosis of a pelvic mass. Gynecol Oncol Rep. 2016;18:29-31.

8. Arnogiannaki N, Grigoriadis C, Zygouris D, Androut- 
sopoulos G, Derdelis G, Terzakis E. Primary ovarian non-Hodgkin's lymphoma. Eur J Gynaecol Oncol. 2011;32(4):441-442.

9. Jina Yoon, Seok Jin Kim, Jonh Ho Won, Chul Won Choi, Hyeon Seok Eom, Jin Seok Kim, Min Kyoung Kim, et al. Clinical features and prognostic relevance of ovarian involvement in Non-Hodgkin's Lymphoma: a consortium for improving survival of lymphoma (CISL) report. Blood. 2009;114(22):4999.

10. Gutierrez-Garcia L, Medina Ramos N, Garcia Rodriguez R, Barber MA, Arias MD, Garcia JA. Bilateral ovarian Burkitt's lymphoma. Eur J Gynaecol Oncol. 2009;30(2):231-233.

11. Rao IS. Role of immunohistochemistry in lymphoma. Indian J Med Paediatr Oncol. 2010;31(4):145-147.

12. Kumar N, Kumar R, Bera A, Srinivasan R, Sharma SC. Primary ovarian lymphoma: a case report and review of literature. J Obstet Gynaecol India. 2014;64(1):65-67.

13. Senol T, Doger E, Kahramanoglu I, Geduk A, Kole E, Yucesoy I, Caliskan E. Five cases of non-hodgkin Bcell lymphoma of the ovary. Case Rep Obstet Gynecol. 2014;2014:392758. 